\title{
Many-body and correlation effects in semiconductors
}

\author{
D. S. Chemla \& Jagdeep Shah $\dagger$
}

* Department of Physics, University of California at Berkeley, and Materials Science Division, Lawrence Berkeley Laboratory, Berkeley, California 94720, USA $\dagger$ Bell Laboratories, Lucent Technologies, Holmdel, New Jersey 07733, USA

Solids consist of $10^{22}-10^{23}$ particles per cubic centimetre, interacting through infinite-range Coulomb interactions. The linear response of a solid to a weak external perturbation is well described by the concept of non-interacting 'quasiparticles' first introduced by Landau. But interactions between quasiparticles can be substantial in dense systems. For example, studies over the past decade have shown that Coulomb correlations between quasiparticles dominate the nonlinear optical response of semiconductors, in marked contrast to the behaviour of atomic systems. These Coulomb correlations and other many-body interactions are important not only for semiconductors, but also for all condensed-matter systems.

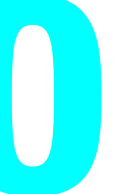

ne of the most fascinating properties of quantum mechanical systems is that they can be in entangled states, that is, a coherent superposition of eigenstates unknown in classical systems. Entangled states have been observed in atomic physics, but they remain elusive in condensed matter. The primary difficulty is that they survive only as long as the coherence is maintained, and numerous processes conspire to produce decoherence, dephasing and relaxation on an extremely short timescale $\left(\sim 10^{-12} \mathrm{~s}\right)$ in condensed matter, which is typically composed of $10^{22}-10^{23}$ particles $\mathrm{cm}^{-3}$ that interact through the infinite-range Coulomb force. The complexity of the problem makes any theoretical approach extremely difficult. About five decades ago, Landau proposed that real particles, strongly interacting among themselves but evolving in the real vacuum, be mapped onto 'quasiparticles'. The quasiparticles are 'dressed' by a part of the interaction, and are relatively long-lived excitations of the many-body system evolving in a 'new vacuum' that consists of the 'rest' of the many-body system and the part of the Coulomb interaction not accounted for in the quasiparticles. The quasiparticles are complex objects (Cooper pairs in a superconductor, excitons in a semiconductor, and so forth), and the new vacuum itself can be structured (it can have an antiferromagnetic order in the copper oxides) and dynamical (it can sustain phonons and magnons). For many solids, the concept of quasiparticles has been very useful for describing the ground state and small departures from it, that is, the most basic excitation and the linear response to weak external perturbations. However, the part of the Coulomb forces not accounted for in the formation of quasiparticles leads to interactions between these quasiparticles, inducing nonlinearities and destroying their phase coherence. Scientists interested in the linear response of a many-body system like to call these interactions 'residual'. They are, however, not small, as shown below. They are residual only in the sense that we do not know (yet) how to treat them correctly. Understanding the influence of these many-body interactions and Coulomb correlations is one of the central challenges of condensed-matter physics.

Semiconductors form an ideal laboratory for quantitatively investigating the role of Coulomb correlations for several important reasons. Their linear properties are well understood because of a wealth of theoretical background and well established approximations that have been developed over several decades, and because of the nearly perfect samples of bulk materials and nanostructures that are now available. Furthermore, the small effective mass and large dielectric constant of semiconductors allow one to explore in 'tabletop' experiments many physical regimes that would require extreme, and often unattainable, conditions in other systems. For example, the exciton Rydberg in the model semiconductor gallium arsenide (GaAs) is only $4.2 \mathrm{meV}$, about 3,000 times smaller than the hydrogen Rydberg $(13.6 \mathrm{eV})$. Consequently, in GaAs the exciton ionization field, about $1 \mathrm{~V} \mathrm{\mu m}^{-1}$, can be easily achieved. Similarly, the magnetic field for which the cyclotron radius is equal to the Bohr radius (about $3.5 \mathrm{~T}$ in GaAs) is easily delivered by commercial magnets, whereas for hydrogen it is about $10^{4} \mathrm{~T}$, and is only found at the surface of neutron stars. Finally, semiconductors are a prototype for many condensed-matter systems so that the information about Coulomb correlations that we can obtain by studying them is valuable in understanding correlations in more complex systems.

Quasiparticles, such as excitons, dominate the linear optical properties of semiconductors near the electronic bandgap. Therefore, interactions between these quasiparticles profoundly influence the nonlinear optical response of semiconductors. In order to understand all the ubiquitous processes associated with Coulomb correlations, it is clear that one must drive the many-body systems away from their ground state and investigate the nonlinear regime. Indeed, a number of investigations during the past decade have unambiguously demonstrated that Coulomb interactions dominate the nonlinear optical response of semiconductors, in contrast to atoms, whose nonlinear response is governed solely by the Pauli exclusion principle. Significant new understanding of the physics of many-body and Coulomb correlation effects have been obtained through time-resolved nonlinear optical spectroscopy. A review of these results, and their implications, is appropriate at this time. Our goal is to emphasize the physical insights recently obtained and to discuss how they can further our understanding of Coulomb correlations in condensed matter in general.

We begin by reviewing two seminal experiments ${ }^{1-3}$ that clearly demonstrate the richness in the nonlinear response of semiconductors, and its fundamental and profound differences from atoms. In particular, the response of semiconductors cannot be understood in terms of the non-interacting two-level model ${ }^{4}$ that has worked so well for dilute atomic systems. We will show that Coulomb interaction is responsible for this difference, and discuss a mean-field formalism, the semiconductor Bloch equations ${ }^{5}$, developed to understand these experiments. In the stationary limit, this meanfield theory shares many features with the Bardeen-CooperSchrieffer (BCS) theory of superconductivity; this shows that laser-excited semiconductors are indeed governed by the same type of interactions as other quantum-coherent many-body systems in condensed matter.

Although the mean-field theory has enjoyed considerable success 
at moderate and high densities, recent studies in regimes of low excitation and very short timescale show that the behaviour of semiconductors is even more complex ${ }^{6,7}$. In these regimes one can probe timescales shorter than the time between quasiparticle collisions so that there are not enough scattering events over the time span of an experiment to establish the mean-field conditions. These investigations, therefore, access regimes where the fluctuations in the scattering between elementary excitations induce large fluctuations of the mean-field order parameters. Then four-particle and higher-order correlations become important and even dominant. Many-particle correlation functions appear in almost all theoretical approaches for describing condensed matter; however, it is usually extremely difficult to access these quantities experimentally. These recent studies ${ }^{6,7}$ allow for the first time the direct investigation of high-order correlations. We will discuss the new insights into many-body physics provided by such experiments.

Finally, Fermi's golden rule and Boltzmann kinetics are two cornerstones of solid-state theory and are the basis for calculating transition and scattering probabilities between two states. They assume that collisions are instantaneous and only the mean free time between the collisions matters. These two cornerstones of solid-state theory are no longer valid on timescales much shorter than the characteristic periods of the entities involved in a 'collision'. The wave-like nature of excitations in solids tells us that all interactions between waves are nothing but interference phenomena, which therefore have a finite duration on the scale of at least one period of the interacting waves. Collisions cannot be considered as 'complete' for times shorter than these periods, and the

\section{Box 1}

\section{Principle of time-resolved nonlinear optical spectroscopy experiments}

The dynamical response of a semiconductor following excitation by an ultrafast laser pulse can be conveniently divided into coherent and incoherent regimes. Timescales for various regimes depend on the nature of quasiparticles and have been discussed earlier ${ }^{14}$. Coulomb correlation effects, the focus of this review, manifest themselves most directly in the coherent response of semiconductors. Four- and six-wave-mixing experiments probe the coherent response (polarization) of the medium, pump/probe experiments investigate both the polarization and the population dynamics, and time-resolved luminescence experiments probe primarily the population or incoherent dynamics.

The generic experimental configuration for time-resolved nonlinear optics experiments is shown in the figure. One, generally weak, laser pulse $\left(E_{1}(t)\right)$ propagating along direction $\bar{k}_{1}$ excites a sample at $t=0$. Alone, this pulse would probe the linear properties of the sample, and hence it is often called the probe pulse. A second laser pulse (the pump pulse), $E_{2}(t)$, propagating along $\bar{k}_{2}$, is delayed and excites the sample at $t=\tau_{\mathrm{d}}$. Because of the nonlinearities in semiconductors the response of the sample to the total field, $E_{\mathrm{T}}(t)=E_{1}(t)+E_{2}(t)$ is not the sum of the responses to each field. In general, two types of measurements can be performed. They are called pump/probe and coherent wave mixing experiments ${ }^{12,14,15}$.

In the first category the small changes in sample transmission, seen by the probe pulse and induced by the pump pulse, are measured. In the small signal regime, the differential transmission spectrum reproduces the changes in the absorption spectrum of the sample induced by the second field. It can be measured for a series of time delays $\tau_{\mathrm{d}}$.

In the second category of experiments, the two fields coherently interfere in the sample and generate polarization waves emitting photons in background-free and momentum-conserving directions (the phase is conserved in a coherent process). For example, at the third order in the laser fields, photons are emitted in the direction $\bar{k}_{\mathrm{FWM}}=2 \bar{k}_{2}-k_{1}$, whereas at the fifth order they are emitted in the direction $\bar{k}_{\mathrm{swm}}=3 \bar{k}_{2}-2 k_{1}$ and so on. Because of the (minimum) number of photons involved in these processes, the experiments are called four-wave-mixing (FWM), six-wave-mixing (SWM) and so on. To understand the physical underpinning of the nonlinearity involved in this process, we can spectrally and/or temporally resolve the coherent wave mixing signals at fixed $\tau_{\mathrm{d}}$. Thus these techniques are called spectrally resolved FWM (or SWM) or time-resolved FWM (or SWM) and so forth. In the case of atomic-like systems the profile of the timeresolved signal is a step function followed by an exponentially decaying tail characterizing the dephasing of the associated polarization. For this reason, the easiest and most commonly used measurement technique is simply to integrate the coherent wave mixing signal with a slow detector as $\tau_{\mathrm{d}}$ is varied. This determines the so-called timeintegrated signal. For atomic systems, it reproduces, as a function of the time delay $\tau_{\mathrm{d}}$, the same behaviour as does the time-resolved signal as a function of the real time $t$.

In addition to measuring the intensity of the coherent response, it may be desirable to obtain information about the phase of the emitted signal. Spectral interferometry provides a means for accomplishing this ${ }^{37}$. In this technique, the unknown emission is collinearly combined with a suitably delayed, well characterized, laser pulse whose spectrum overlaps that of the unknown emission, and the spectra of the unknown, the laser and the combined beams are measured. A spectral interferogram, obtained by subtracting the two individual spectra from the combined spectrum, exhibits spectral fringes corresponding to the time delay. Fourier transform analyses of the interferogram allow us to determine the amplitude and the phase of the unknown signal in the temporal and spectral domains. The results can be plotted in a Wigner-function representation.

In contrast to transport experiments that probe equilibrium properties averaged over many parameters, ultrafast nonlinear optical experiments allow us to probe a highly nonequilibrium regime, and provide information not possible through other means.

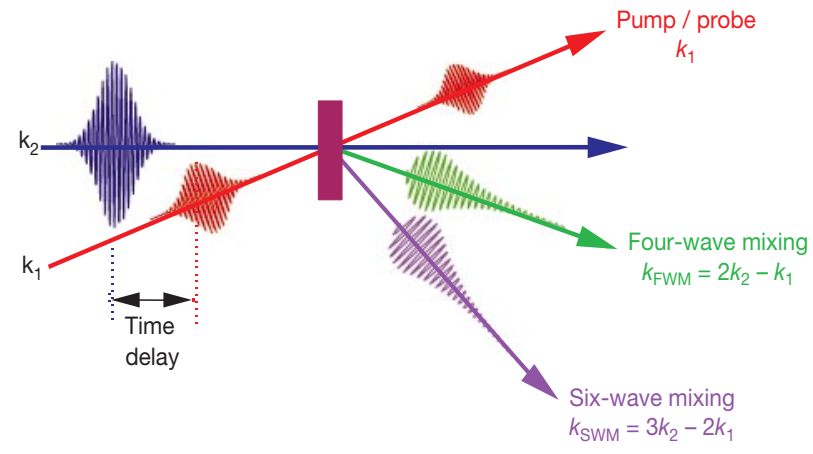

Box 1 Figure Schematic of a generic time-resolved nonlinear optical experiment. 
formalism of quantum kinetics instead of Boltzmann kinetics must be used. We will present examples of quantum kinetic effects in semiconductors, and show that collision in progress can in fact be reversed under appropriate conditions ${ }^{8}$. We note that intracollisional field effects have been considered quite extensively in theoretical quantum transport studies (see, for example, ref. 9). Therefore, the insights obtained from the optical studies reviewed here are also relevant to transport phenomena ${ }^{10}$.

A large number of researchers have contributed to the advances in this field. However, this review cannot be comprehensive, and thus we limit ourselves to a broad overview of the most significant developments. The reader is also referred to a number of excellent recent review articles and books $s^{5,11-18}$.

\section{Nonlinear optical response of semiconductors}

The interaction between quasiparticles in semiconductors is best investigated by studying their coherent response. Box 1 summarizes the experimental techniques generally used for such studies and discusses the behaviour of dilute atomic systems in such experiments ${ }^{14}$. Here we discuss two seminal experiments demonstrating that the nonlinear response of semiconductors is very different from that of a non-interacting two-level system.

In the first experiment the time-integrated four-wave mixing (FWM) response of a high quality GaAs quantum-well sample was measured as a function of the lattice temperature ${ }^{1}$ with a time resolution of about $300 \mathrm{fs}$. Figure 1 shows the measured timeintegrated signal in the $2 \bar{k}_{2}-\bar{k}_{1}$ direction as a function of time delay $\tau_{\mathrm{d}}$ between the pulses. $\tau_{\mathrm{d}}$ is considered positive (negative) when pulse 1 (2) precedes pulse 2 (1). Figure 1 shows that a strong signal was observed for negative time delays $\left(\tau_{\mathrm{d}}<0\right)$ much larger than the time resolution of the experiment. This result is in striking

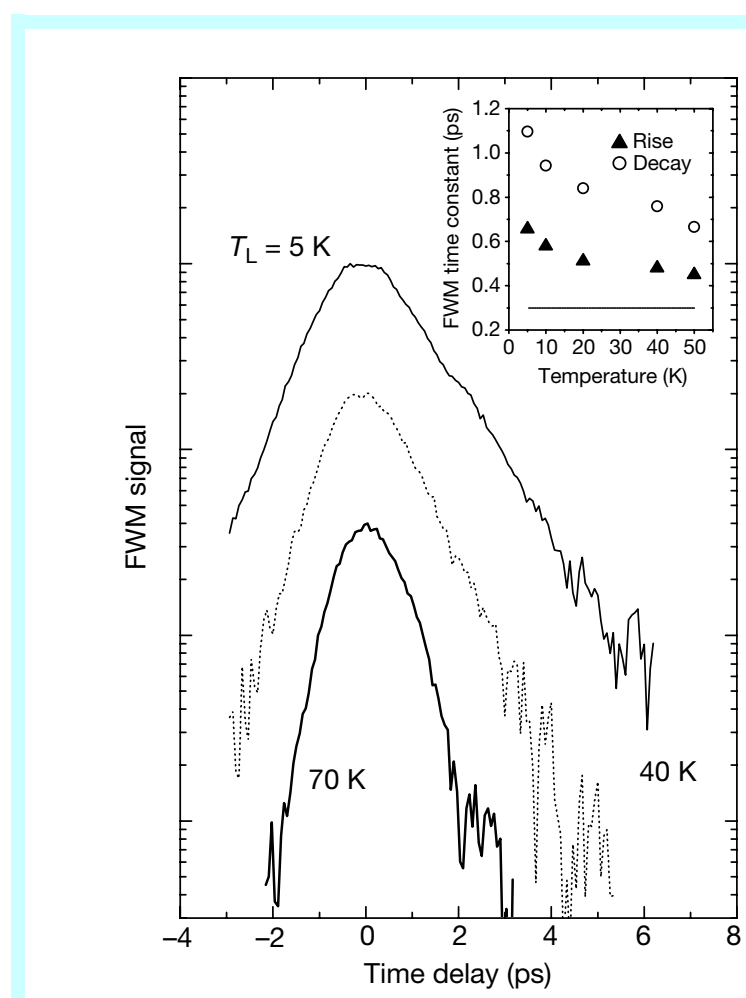

Figure 1 Strong negative time-delay signals in time-integrated four-wave mixing (FWM) from GaAs quantum wells. Time-integrated FWM signals in the $2 \bar{k}_{2}-\bar{k}_{1}$ direction versus time delay $\tau_{\mathrm{d}}$ between the two pulses for a $170 \AA$ GaAs multiplequantum-well sample at three different temperatures. The results show an unexpected signal at negative time delays much longer than the system time resolution. qualitative contrast to the expectation of no FWM signal for $\tau_{\mathrm{d}}<0$ (ref. 4) for non-interacting independent two-level systems, for example atoms (see Box 1). The time constant for the rise of the signal is half that for the decay (inset, Fig. 1). These observations provided the first evidence that the nonlinear response of semiconductors is very different from that of atoms.

Time-resolved four-wave mixing experiments showed further surprising results ${ }^{2,3}$. For atomic systems, the time-resolved signal in the $2 \bar{k}_{2}-\bar{k}_{1}$ direction is expected to peak when pulse 2 reaches the sample, and then decay exponentially with time $t$. Figure 2 shows the time-resolved FWM signal in the $2 \bar{k}_{2}-\bar{k}_{1}$ direction from a highquality GaAs quantum-well sample, with $t=0$ corresponding to the arrival of pulse 2 at the sample. In contrast to the expectations for two-level atoms, the signal peaks at time $t=t_{0}$, where $t_{0}$ is extremely long (as much as 10 times the laser pulse width) $)^{2}$. The delay decreases strongly as the dephasing rate decreases with increasing temperature (Fig. 2), but was shown to be independent of $\tau_{\mathrm{d}}$, in contrast to the behaviour expected for the photon echo signal from inhomogeneous systems ${ }^{14}$. The time-integrated signal at negative time delays, and the delayed peak in the time-resolved signals were explained in terms of local fields resulting from Coulomb interactions ${ }^{19}$. A more detailed discussion of the physical origins of these effects is given in the next section.

These two experiments led to a flurry of activity, both theoretical and experimental, aimed at understanding the nonlinear response of semiconductors. A review of the early work can be found in refs 20 and 21 .

\section{The semiconductor Bloch equations}

The material parameters that enter the Maxwell equations and describe nonlinear response are the polarization and the carrier density (population). In the density matrix formalism ${ }^{4}$, they represent the off-diagonal and diagonal components of the density matrix, respectively. In general, they are given by the expectation

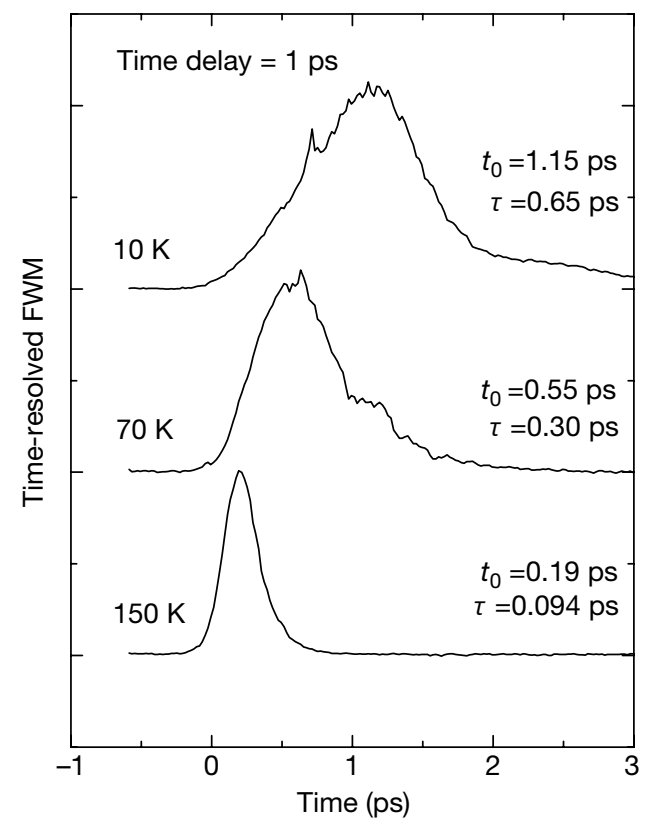

Figure 2 Delayed rise of FWM signals from GaAs quantum wells. Temporal evolution of FWM signal from a nearly homogeneously broadened $170 \AA$ GaAs multiplequantum-well sample showing the unexpectedly slow rise of the signal; the peak occurs at shorter times as the dephasing rate increases with increasing temperature ${ }^{2}$. $t_{0}$ is defined in the text; $\tau$ is the decay time constant. 
value of products of two creation and destruction operators. Semiconductors are different from an ensemble of two-level systems because Coulomb interactions between electrons and/or holes introduces products of four creation and destruction operators in the equations of motion of these products (see Box 2). This results in an infinite hierarchy of coupled differential equations for products of 2, 4, $6 \ldots$ operators that cannot be solved in general. This 'complexity disaster' appears in all many-body formalisms: the
Feynman diagrams, Green's functions and generalized density matrix approach. A consistent truncation scheme is necessary to provide a description at an appropriate level of accuracy. Because of the complexity of the problem, however (see Box 2), the input of experiment is crucial in guiding the theorists to identify the dominant processes. An approach able to account for the lowestorder correlation, first introduced to describe the excitonic optical Stark effects ${ }^{22}$, has been developed ${ }^{23}$. It is a mean-field theory based

Box 2

Many-body effects in light-semiconductor interaction

Here we give a very basic description of the main issues of condensedmatter many-body theory. The reader interested in going beyond this (incomplete and intuitive) description can refer to refs 5, 12, 13, 15, 18 and 28-31.

The hamiltonian of a semiconductor is governed by electromagnetic interaction only. The Coulomb interaction among electrons and nuclei can be condensed in the 'effective mass approximation' in which the electronic levels are described by a few energy bands. The real electrons are mapped onto quasiparticles (unfortunately also called 'electrons') with an effective mass ( $m \rightarrow m^{*} \ll m$ ) close to the band extrema, a dispersion $\hat{\epsilon}(k)$ far from these special points, and a Coulomb interaction screened by the high-frequency dielectric constant (about 10-15 in most semiconductors).

Very often, because of doping with impurities or optical excitation, a relatively small number (compared to $10^{23} \mathrm{~cm}^{-3}$ ) of electrons are present in the conduction bands and a few electrons are 'missing' in the valence bands. It has become customary to introduce yet another quasiparticle, the holes, to describe these missing electrons.

With all these caveats, the electronic part of the semiconductor hamiltonian is written in a simplified form

$$
H_{\mathrm{sc}}=H_{\mathrm{e}}+H_{\mathrm{h}}+H_{\mathrm{e}-\mathrm{e}}+H_{\mathrm{h}-\mathrm{h}}+H_{\mathrm{e}-\mathrm{h}}
$$

with

$$
H_{\mathrm{e}}=\sum_{\alpha, k} \epsilon_{\alpha}(k) \hat{e}_{\alpha, k}^{+} \hat{e}_{\alpha, k}
$$

and

$$
H_{\mathrm{h}}=\sum_{\beta, k} \epsilon_{\beta}(k) \hat{h}_{\alpha, k}^{+} \hat{h}_{\alpha, k}
$$

Here $\hat{e}_{\alpha, k}^{+}, \hat{h}_{\alpha, k}^{+}, \hat{e}_{\alpha, k}$ and $\hat{h}_{\alpha, k}$ are the creation and destruction operators for electrons (e) or holes (h) with wave-vector $k$ in the band $\alpha$, whose energy $\epsilon_{\alpha}(k)$ is described by the proper effective mass expressions. Close to the band extrema these can be approximated by parabolas, $\epsilon_{\alpha}(k)=\hbar^{2} k^{2} / 2 m_{\alpha}^{*}$, characterized by the effective masses $m_{\alpha}^{\star}$.

The last three terms of the hamiltonian describe, respectively, the Coulomb scattering between electrons, between holes and between electrons and holes. They have the generic form

$$
H_{\mathrm{e}-\mathrm{e}}=\sum_{\alpha \alpha^{\prime} \beta \beta^{\prime}, k k^{\prime}} v_{q} \hat{e}_{\alpha, k+q}^{+} \hat{e}_{\beta, k-q}^{+} \hat{e}_{\alpha^{\prime}, k}^{+} \hat{e}_{\beta^{\prime}, k}^{+}
$$

They contain products of four creation and destruction operators and correspond to the fundamental Feynman diagram shown in panel $\mathbf{a}$ of the figure.

In the semi-classical approximation (the electromagnetic field (EMF) is not quantized, because the intense laser pulses contain a very large number of photons) the interaction with the light field is given by the sum of Rabi frequencies

$$
H_{\mathrm{SC}-\mathrm{EMF}}=-\sum_{\alpha \beta, k} \mu_{\alpha \beta, k} E(t) \hat{e}_{\alpha, k}^{+} \hat{h}_{\beta,-k}^{+}+h \mathrm{C}
$$

$H_{\text {SC-EMF }}$ describes the creation and destruction of electron-hole pairs by absorption and emission of photons. The coupling with the electromagnetic field, $E(t)$, is measured by the dipole matrix elements $\mu_{\alpha \beta, k}$ for interband, $\alpha \neq \beta$, or intraband, $\alpha=\beta$, transitions and is illustrated in panel $\mathbf{b}$ of the figure.

We note the analogy between panel $\mathbf{b}$ and 'half' of panel $\mathbf{a}$. Indeed, they both describe the scattering of particles from one state to another. The main difference, however, is that the photons correspond to the applied transverse field $E(t)$, whereas the Coulomb potential describes the mutual longitudinal field exercised by the particles on one another.

Fermion operators always appear in pairs. So when we try to describe the time evolution of the product of an even number $(2 n)$ of such operators under a hamiltonian containing $H_{\mathrm{SC}}$ we obtain equations involving at least $(2 n+2)$ operators, and that triggers the generation of an infinite number of coupled equations. This process can take different embodiments depending on the formalism used (Feynman diagram, Green's function, density matrix), but the root cause is always the same. We note that the correlation functions that appear in the theory describe properties of the electronic system as well as the scattering processes that can happen in the interacting many-body system. For example, the electron and hole populations

$$
n_{e, \alpha}(k)=\left\langle\hat{e}_{\alpha, k}^{+} \hat{e}_{\alpha, k}\right\rangle \text { and } n_{h, k}=\left\langle\hat{h}_{\alpha, k}^{+} \hat{h}_{\alpha, k}\right\rangle
$$

and the transition amplitudes

$$
p_{\alpha \beta}(k)=\left\langle\hat{e}_{\alpha, k}^{+} \hat{h}_{\beta,-k}^{+}\right\rangle
$$

are 2-particle correlation functions: respectively, the diagonal and offdiagonal elements of the density matrix. Higher-order correlation functions often have an intuitive interpretation; in the context of this review, for example, the 4-particle correlation function, $\left\langle\hat{e}_{\alpha, k}^{+} \hat{h}_{\beta,-k}^{+} \hat{e}_{\alpha^{\prime}, k^{\prime}}^{+} \hat{h}_{\beta^{\prime},-k^{\prime}}^{+}\right\rangle$, describes the creation of two electron-hole pairs in a two-photon transition.
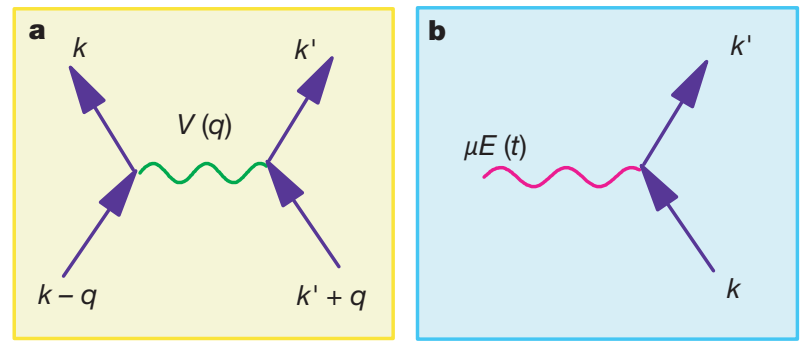

Box 2 Figure Feynman diagrams for (a) Coulomb interaction between carriers, and (b) coupling with electromagnetic field. 
on the random phase approximation (RPA $)^{24}$, which is founded on the physical argument that under suitable circumstances a sum of exponential terms with randomly varying phases may be neglected. (RPA goes by many names, including time-dependent HartreeFock approximation.) RPA results in the factorization of the expectation value of the four-particle operators into the products of 2-particle operators. Within this theory, the time evolution of the polarization and density form a closed system, the semiconductor Bloch equations $(\mathrm{SBE})^{5}$. We use this formalism here because the SBE are the semiconductor analogue of the optical Bloch equations (OBE) that are extensively used in atomic and molecular physics. The OBE are themselves the optical analogue of the equations first developed by Bloch $^{25}$ for the spin systems.

The details of the two seminal experiments discussed in the previous section can be described accurately by numerical solution of the SBE. However, the underlying physics can be qualitatively understood by examining the form of these equations. The SBE are directly analogous to the OBE except that the effective Rabi frequency (describing the light-matter coupling) and the energy of the quasiparticles (for example, excitons) are renormalized (that is, modified) by polarization and population respectively. Very intuitively these 'renormalizations' can be explained by saying that the charges in the medium feel not only the applied laser field, but also the field of all the other dipoles induced in the semiconductor. Similarly, their energy is modified by the Coulomb interaction with the other induced charges. These renormalizations profoundly influence the nonlinear response of semiconductors ${ }^{15,26,27}$. For non-interacting two-level systems, the FWM signal appears as an

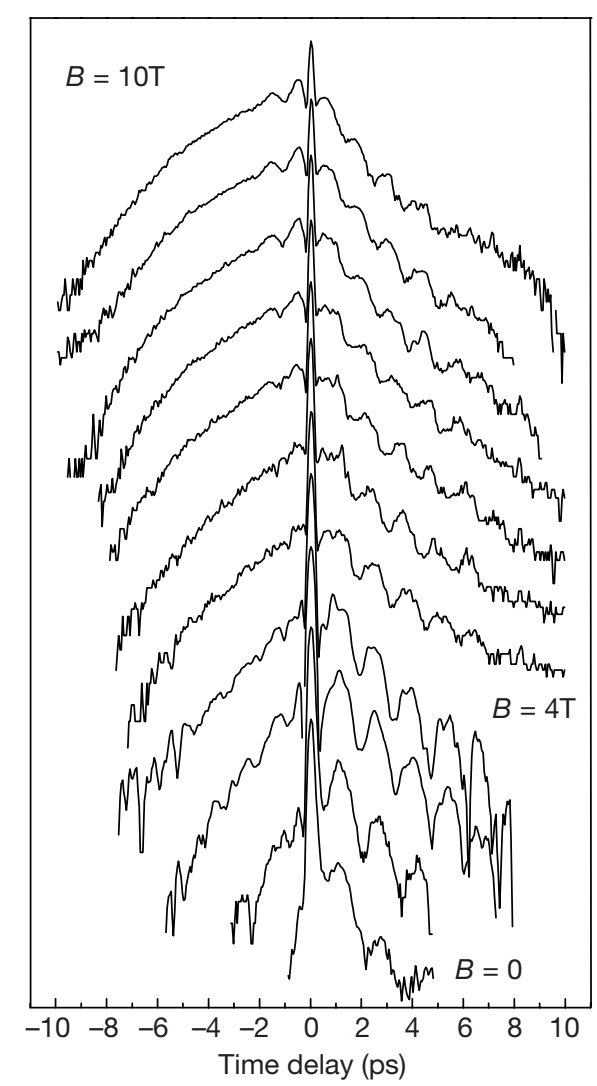

Figure 3 FWM signals due to high-order correlation in GaAs. Time-integrated FWM signal from bulk GaAs (log scale) at $1.8 \mathrm{~K}$ for various magnetic fields. The curves are displaced vertically for clarity. We note the huge increase in the negative time-delay component with increasing $B$ (ref. 39). interaction between the field of the second pulse with the polarization created by the first pulse and thus does not exist when they do not overlap $\left(\tau_{\mathrm{d}}<0\right)$. In a semiconductor, the renormalization of the Rabi frequency allows interaction between the polarizations created by the two pulses. Because polarizations persist for times of the order of the dephasing time after the exciting electric field, they overlap regardless of the pulse sequence and produce the $\tau_{\mathrm{d}}<0$ signal. Furthermore, these renormalizations allow diffraction of polarization. The third-order polarization resulting from diffraction of polarization increases with time and reaches a peak at a time determined by the dephasing time. Therefore, the time-resolved signal exhibits a delayed peak after the second pulse, as observed experimentally.

Recent results have shown that the nonlinear response of semiconductors is even more complex, and higher-order, 4-particle and 6-particle correlations make important and sometimes dominant contributions to the nonlinear response. These are discussed in the next section.

\section{High-order correlation effects}

Although the mean-field theory (SBE) has been extremely successful at high and moderate densities, at low densities we can investigate timescales that are short compared to the time for one quasiparticle to interact with a substantial fraction of its neighbours. Thus it becomes possible to observe deviations from mean-field theory, a regime where high-order correlations become dominant. Indeed, recent comparative experimental and theoretical studies have revealed features related to genuine 4-particle and 6-particle correlations. Different ways of extending the theory beyond the 2-particle, mean-field approach of SBE have been developed $^{12,17,18,28-31}$. Some experimental investigations of higher-order correlations $s^{6,7,32-34}$ are discussed below.

The effects of 4-particle correlations in the continuum of exciton scattering states are shown in Fig. 3 where time-integrated FWM measured in a GaAs sample with heavy-hole and light-hole exciton resonances in the absorption spectrum is shown for magnetic field varying from 0 to $10 \mathrm{~T}$ (ref. 6). The signal has an expected profile for $\tau_{\mathrm{d}}>0$ : beats between the two exciton resonances are superimposed on an exponential decay, with only slight dependence on $B$. In contrast, the Coulomb-induced signal for $\tau_{\mathrm{d}}<0$ changes drastically as $B$ increases. Not only does its magnitude increase, but also its profile becomes highly non-exponential with an unusual positive curvature and extends as far as $\tau_{\mathrm{d}} \approx 10 \mathrm{ps}$, or 100 times the duration of the laser pulse (compare with Fig. 1). The explanation of this effect is that the excitons created in the semiconductor by one laser pulse are very strongly distorted by the magnetic field. Therefore, they acquire a quadrupole moment and through the Coulomb interaction they generate a coherent 4-particle correlation in the medium. It corresponds to a two-photon active coherence ${ }^{35}$ and, therefore, cannot deliver one photon emission by itself. Thus it is stored in the medium until the second pulse triggers that emission. This 'coherent memory' can be interpreted as a non-markovian process involving the 2-particle correlation polarization waves interacting with a bath of 4 -particle correlations ${ }^{36}$.

The effects of 4-particle correlations have been shown to be important in other recent experiments as well ${ }^{7}$. At low densities, incident radiation resonant with excitons in quantum wells is scattered (resonant Rayleigh scattering) due to disorder (and hence inhomogeneous broadening of excitons) at low exciton densities. However, 4-particle correlations can lead to intrinsic resonant Rayleigh scattering even in the absence of disorder. Spectral interferometry ${ }^{37,38}$ allows determination of the temporal dynamics and the spectra of the amplitude and the phase of coherent radiation. Four-particle correlations can be thought of as the bound and unbound (continuum) states of biexcitons (an entity composed of two excitons). New spectral features related to 4particle correlations have been identified recently in resonant 
Rayleigh scattering from high-quality GaAs quantum wells ${ }^{38}$. The Wigner function provides a convenient means of displaying emitted field. The coherent spectrum and dynamics can be deduced from such a representation (Fig. 4). The coherent spectrum deduced from such measurements shows not only the disorder-induced resonant Rayleigh scattering from excitons (near $807 \mathrm{~nm}$ ) but also intrinsic Rayleigh scattering from the bound state of the biexciton (near $808 \mathrm{~nm})$ and the continuum 4-particle states $(<807 \mathrm{~nm})$. The dip in the response at a wavelength slightly below $807 \mathrm{~nm}$ is due to interference between the response from excitons and 4-particle correlations. These experiments provide the first information on the spectrum of 4-particle correlations, and have the potential of providing new understanding of the nature of 4-particle correlations.

Higher-order correlations such as six-particle correlation effects are difficult to identify in conventional FWM and pump/probe experiments. However, six-wave-mixing (SWM) experiments are expected to be more sensitive to the high-order correlations, although 2-particle and 4-particle correlations also contribute to the SWM signals. Indeed, recent comparative experimental and theoretical studies ${ }^{32}$ have revealed genuine 6-particle correlations in a single $\mathrm{ZnSe}$ quantum well. Figure 5 a presents the contour maps of the spectrally resolved SWM emission measured in the direction $3 \bar{k}_{2}-2 \bar{k}_{1}$ for incident laser pulses polarized along the $x$ and $y$ axes, and the signal polarized along the $y$ axis. These plots give information both on the dependence of the signal at a given frequency on the time delay $\tau_{\mathrm{d}}$, and dependence on real time at a given $\tau_{\mathrm{d}}$ (related to the Fourier transform of the frequency dependence at a given $\tau_{\mathrm{d}}$ ). Two contributions are seen at the exciton and the exciton-biexciton energies, both decaying smoothly without temporal or spectral beats. Figure $5 b-d$ shows theoretical modelling of the experiments at three levels of sophistication. Figure $5 \mathrm{~b}$ accounts only for the 2particle and 4-particle correlation involved in the purely coherent emission, that is, 1-photon and 2-photon coherence. Figure $5 \mathrm{c}$ accounts in addition for incoherent exciton densities and Fig. $5 \mathrm{~d}$ also accounts for 6-point correlations that describe transitions from incoherent exciton densities to two-pair states; these are often referred to as 'excited-state absorption' in atomic physics. The theory plots are obtained by exactly solving numerically the coupled

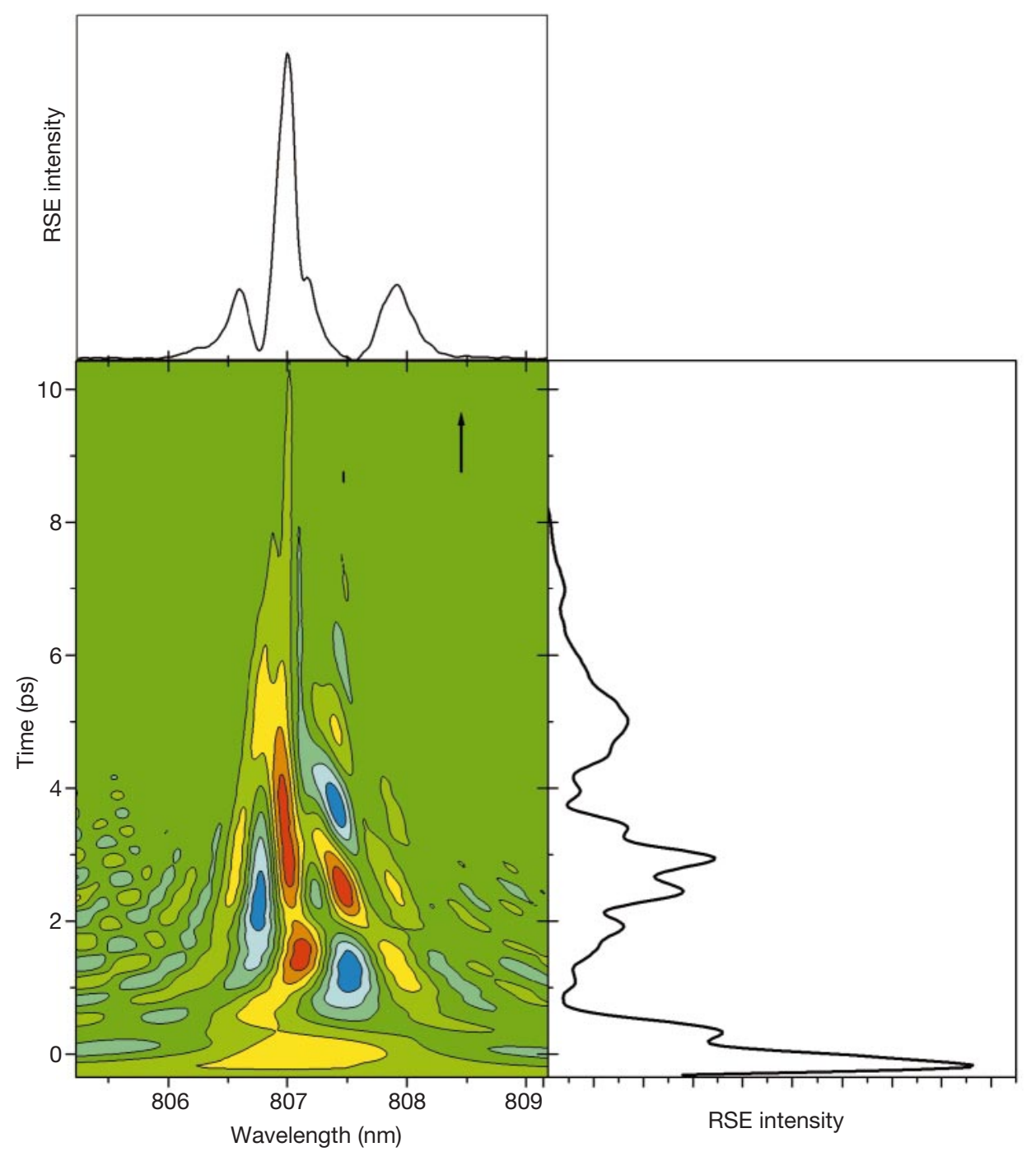

Figure 4 Wigner-function representation of resonant Rayleigh scattering from GaAs quantum wells. Wigner-function representation of the intrinsic and disorder-induced resonant Rayleigh scattering signal from a $130 \AA$ GaAs multiple quantum-well sample at $10 \mathrm{~K}$ at intermediate excitation density. The coherent spectrum and the coherent dynamics can be determined from the Wigner function, as shown. The coherent spectrum shows features related to the spectral response of 4-particle correlations ${ }^{7}$. RSE, resonant secondary emission. 
equations of motion of the 2-, 4- and 6-particle correlation functions and thus the results include the contributions of all orders in the exciting laser field. Figure $5 b$ and $c$ show large disagreements with experiment. But adding 6-point correlations (Fig. 5d) brings the theory into good qualitative agreement with experiment, showing that correlation functions at least of the sixth order are absolutely necessary to describe accurately the SWM experiments.

Although high-order correlation functions appear very often in the theory of many-body effects in condensed matter, they are rarely accessible to direct measurement. The experiments of the type described above provide a means of observing the effects of higher-order correlations and investigating their properties. For example, the dephasing of 4-particle correlation functions by phonons and carrier scattering has been studied recently for the first time ${ }^{39}$

\section{Coherent control, memory effects and relaxation}

Another regime where traditional assumptions fail is that of strongly correlated dynamics where it is no longer justified to treat the interaction between quasiparticles as scattering events local in space and time (a key assumption of the Boltzmann theory). In that case memory effects become observable. In principle, for any quantum mechanical system described by a Schrödinger equation, the knowledge of the hamiltonian and the present state is enough to determine the future evolution. In practice, however, we do not know how to solve the time-dependent Schrödinger equation of complex many-body systems. So it is customary to divide the total system into a subsystem that we are interested in, and the thermal 'bath' of all the other degrees of freedom (reservoir) on which we have only partial information. Memory effects and dissipation occur through the following mechanism: at a time $t^{\prime}$ an interaction occurs between the subsystem and some degrees of freedom of the reservoir, which then evolve with their own dynamics and at a later time, $t$, interact again with the subsystem. From the viewpoint of the evolution of the subsystem, processes depending on the time difference $\left(t-t^{\prime}\right)$ are occurring and hence the subsystem develops a 'memory'. Because the reservoir is so large and contains so many degrees of freedom, in general there are many complex interferences between $t$ and $t^{\prime}$ so that information is lost and, again from the viewpoint of the subsystem, there is 'dissipation'. However, on a timescale that is short compared to the periods of the reservoir excitation and before the reservoir/subsystem scattering processes have randomized the degrees of freedom, the details of the evolution of the coupled system (subsystem plus reservoir) must be accounted for and the dynamics of the subsystem must be described by quantum kinetics, with non-markovian statistics as we mentioned in the introduction. The main relaxation mechanisms in semiconductors are electron-electron and phonon-electron scattering. The timescale of the initial phononelectron non-markovian regime can be estimated by noting that the time it takes a lattice to react to a perturbation is of the order of one period of its oscillation, that is, in the case of the longitudinal optical (LO) phonon in GaAs, $T_{\mathrm{LO}}=115 \mathrm{fs}$.

Memory effects in the nonlinear response of semiconductors have indeed been observed ${ }^{8,34,40-44}$. Here we first discuss non-markovian effects in the coupling of an LO phonon with interband polarization observed in bulk GaAs by FWM experiments using pulses of about $14 \mathrm{fs}$, about eight times shorter than the LO-phonon period ${ }^{8}$. Time-integrated FWM exhibits an oscillatory modulation superimposed on the usual exponential decoherence decay (Fig. 6; curves 7 to 10 ). The period, about $98 \mathrm{fs}$, corresponds to the separation between conduction band states coupled by one LO-phonon. These oscillations are not due to emission or absorption of phonons but correspond to a coherent memory stored in the LO-phonon bath that modulates the interband transition. This phonon-electron interaction, however, is not 'irreversible'. Like any other quantum mechanical 'collision' it is an interference that can be reversed or enhanced by a second perturbation whose phase is appropriately chosen. This reversal was indeed experimentally demonstrated, as shown in Fig. 6. The detailed theory of these experiments is not yet available, but they demonstrate that by using coherent control of the excitation it is possible to manipulate the so-called 'irreversible' scattering processes.

In some special systems it is known from transport experiments that electron-electron interaction can be very significantly reduced. For example, under high magnetic field a two-dimensional electron gas (2DEG) enters the regime of the quantum Hall effect (QHE) and at integer filling factors behaves as an incompressible fluid with a
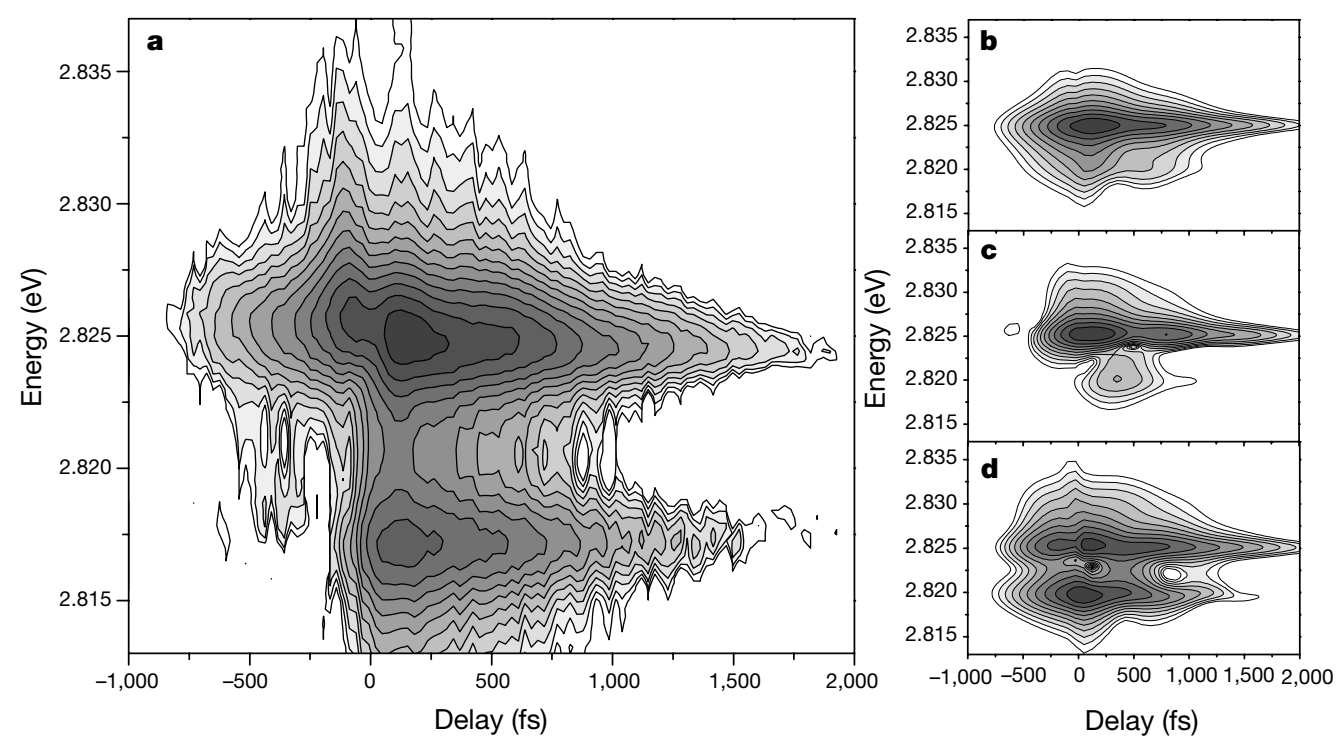

Figure 5 Signature of higher-order correlations in the coherent response of ZnSe. Contour map of spectrally resolved six-wave-mixing (SWM) signal as a function of time delay for a ZnSe quantum well. a, Experiment, with greyscale representing variation of the SWM signal over three orders of magnitude; $\mathbf{b}-\mathbf{d}$, calculated SWM for three different cases discussed in the text ${ }^{32}$. 


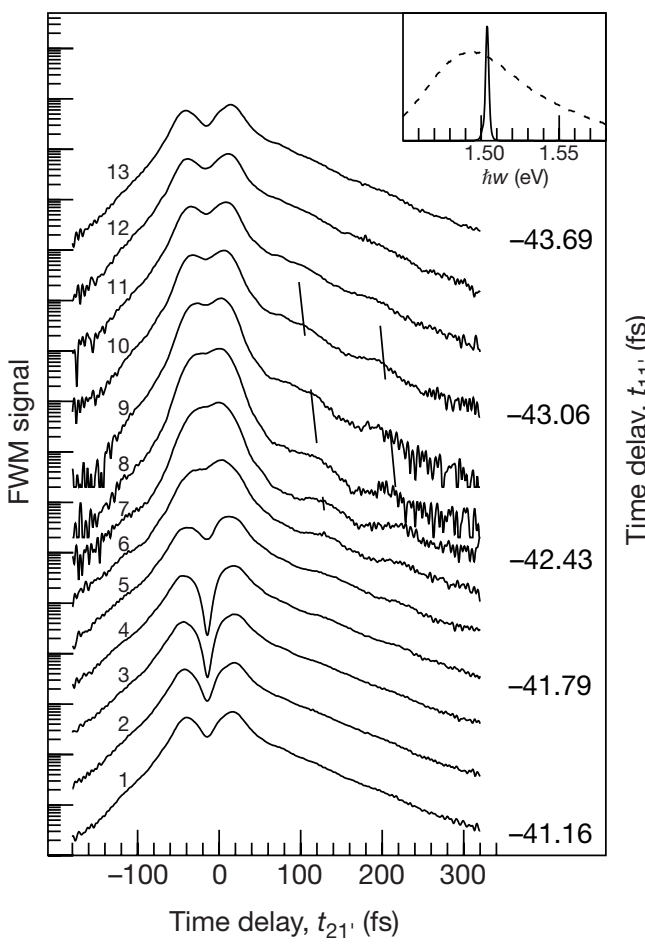

Figure 6 Reversible electron-photon scattering in GaAs. Time-integrated FWM signal from bulk GaAs at $77 \mathrm{~K}$ obtained by using two phase-locked pulses (1 and $1^{\prime}$ ) along $\bar{k}_{1}$ and a single pulse (2) along $\bar{k}_{2}$. The FWM signals are displayed as a function of timedelay $t_{21}$, for many different delays $t_{11}$. The curves are displayed vertically for clarity. The inset shows the exciton and laser spectra ${ }^{8}$.

phase coherence time that diverges at low temperature. One thus expects that the quantum coherence of the interband polarization will exhibit peculiar properties under these conditions. Such behaviour has been observed recently ${ }^{45}$ in FWM experiments performed on 2DEG in the QHE regime, where the laser pulses were tuned to excite electrons into only the highest partially occupied Landau level containing the Fermi energy. At high magnetic field when the Landau levels are only fractionally filled, the time-integrated FWM signal has a complicated profile with a non-exponential behaviour and a long decay time for short time delays. This profile evolves to a single exponential with an unusually long decay time when the Landau level approaches full filling, but the dephasing time experiences a very sudden decrease when the Fermi energy moves to the next Landau level. A measure of the interband polarization dephasing time is obtained by extracting an overall decay time from the FWM profiles and is displayed in Fig. 7 (squares) for two samples with different doping. We note the very large jump each time the system passes through even filling factors for samples with very different densities, showing that this is an effect of the cold 2DEG. When the Landau levels are partially filled, the dephasing originates mainly from the scattering of the photoexcited carriers with the intra-Landau-level collective excitations of the strongly correlated two-dimensional electron liquid. A model based on scattering with the 2DEG magneto-rotons ${ }^{48}$ accounts qualitatively for the salient features seen experimentally as shown in Fig. 7 (circles).

\section{Future directions}

The dominant role of Coulomb correlations in the nonlinear response of semiconductors is now well established. Understanding higher-order correlations in semiconductors remains a challenge
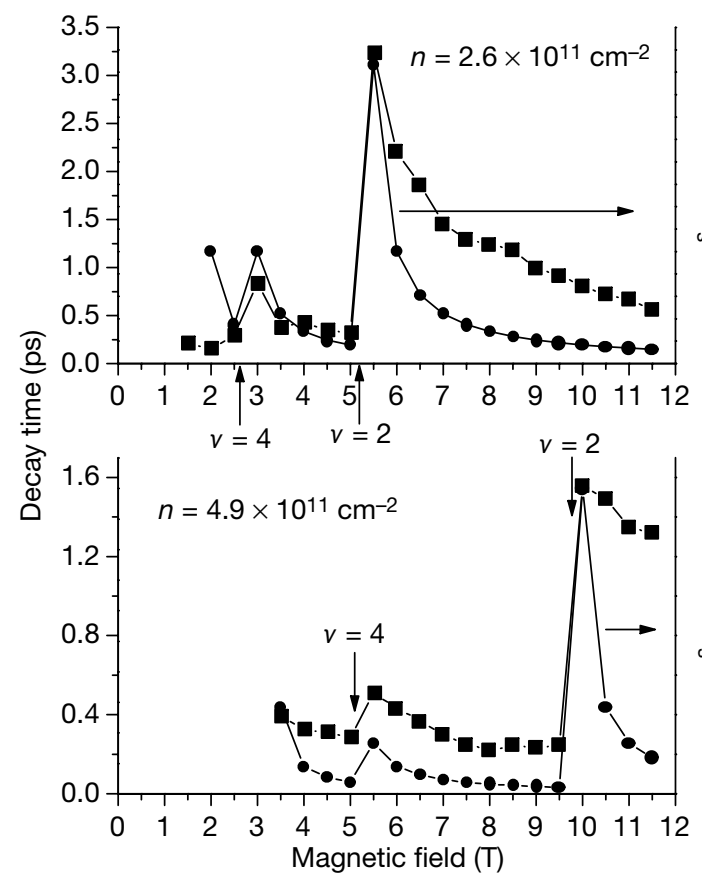

Figure 7 Coherent response of GaAs in the quantum Hall regime. Decay time (squares) of the time-integrated FWM signals from the electron gas in two modulationdoped GaAs quantum-well samples in the fractional quantum Hall regime at $2 \mathrm{~K}$ as a function of the magnetic field that changes spacings between Landau levels and hence the fractional occupancy of Landau levels. The circles correspond to $1 / N_{s}=\nu /[2(N+1)-\nu]$, where $N$ is the Landau level number and $\nu$ is the filling factor $^{45}$

and is a good direction for future investigations. We believe that an even more important challenge is to relate this improved understanding of Coulomb correlations in semiconductors to strongly correlated solids, and investigate whether some of the approaches that have been so fruitful in semiconductors may lead to a better understanding of these solids. High- $T_{\mathrm{c}}$ superconductors, transition metal oxides and polymers are some examples of highly correlated systems. Some work in this direction has already begun ${ }^{46,47}$.

1. Leo, K. et al. Effects of coherent polarization interactions on time-resolved degenerate four-wave mixing. Phys. Rev. Lett. 65, 1340-1343 (1990).

2. Kim, D. S. et al. Unusually slow temporal evolution of femtosecond four-wave-mixing signals in intrinsic GaAs quantum wells: direct evidence for the dominance of interaction effects. Phys. Rev. Lett. 69, 2725-2728 (1992).

3. Weiss, S., Mycek, M.-A., Bigot, J.-Y., Schmitt-Rink, S. \& Chemla, D. S. Collective effects in excitonic free induction decay: do semiconductors and atoms emit coherent light in different ways? Phys. Rev. Lett. 69, 2685-2689 (1992).

4. Allen, L. \& Eberly, J. H. Optical Resonance and Two-Level Atoms (Wiley Interscience, New York, 1975).

5. Haug, H. \& Koch, S. W. Quantum Theory of the Optical and Electronic Properties of Semiconductors (World Scientific, Singapore, 1993).

6. Kner, P., Bar-Ad, S., Marquezini, M. V., Chemla, D. S. \& Schäfer, W. Magnetically enhanced excitonexciton correlations in semiconductors. Phys. Rev. Lett. 78, 1319-1322 (1997).

7. Birkedal, D., Shah, J. \& Pfeiffer, L. N. Coherent secondary emission from resonantly excited twoexciton states: An intrinsic phenomenon. Phys. Rev. B 60, 15585-15588 (1999).

8. Wehner, M. U., Ulm, M. H., Chemla, D. S. \& Wegener, M. Coherent control of electron-LO-phonon scattering in bulk GaAs. Phys. Rev. Lett. 80, 1992-1995 (1998).

9. Rossi, F., Brunetti, R. \& Jacoboni, C. in Hot Carriers in Semiconductor Nanostructures: Physics and Applications (ed. Shah, J.) 153-188 (Academic, Boston, 1992).

10. Haug, H. \& Jauho, A. P. Quantum Kinetics in Transport and Optics of Semiconductors (ed. Klitzing, K. V.) (Springer Series in Solid-State Sciences, Springer, Berlin, 1996).

11. Steel, D. G., Wang, H., Jiang, M., Ferrio, K. B. \& Cundiff, S. T. in Coherent Optical Interactions in Solids (ed. Phillips, R. T.) 157-180 (Plenum, New York, 1994).

12. Mukamel, S. Principles of Nonlinear Optical Spectroscopy (Oxford Univ. Press, New York, 1995).

13. Binder, R. \& Koch, S. W. Nonequilibrium semiconductor dynamics. Prog. Quant. Electron. 19, 307462 (1995).

14. Shah, J. Ultrafast Spectroscopy of Semiconductors and Semiconductor Nanostructures (Springer, Heidelberg, 1999). 
15. Chemla, D. S. in Nonlinear Optics in Semiconductors (ed. Garmire, E. \& Kost, A. R.) 175-256 (Academic, New York, 1999).

16. Khitrova, G., Gibbs, H. M., Jahnke, F., Kira, M. \& Koch, S. W. Nonlinear optics of normal-modecoupling in semiconductor microcavities. Rev. Mod. Phys. 71, 1591-1639 (1999).

17. Perakis, I. E. \& Shahbazyan, T. V. Canonical transformation approach to the ultrafast nonlinear optica dynamics of semiconductors. Int. J. Mod. Phys. B 13, 869-893 (1999).

18. Perakis, I. E. \& Shahbazyan, T. V. Many-body correlation effects in the ultrafast non-linear optical response of confined Fermi seas. Surf. Sci. Rep. 40, 3-74 (2000).

19. Wegener, M., Chemla, D. S., Schmitt-Rink, S. \& Schäfer, W. Line shape of time-resolved four-wave mixing. Phys. Rev. A 42, 5675-5683 (1990).

20. Henneberger, F., Schmitt-Rink, S. \& Göbel, E. O. (eds) Optics of Semiconductor Nanostructures (Akademie, Berlin, 1993).

21. Phillips, R. T. (ed.) Coherent Optical Processes in Semiconductors (Plenum, New York, 1994).

22. Schmitt-Rink, S. \& Chemla, D. S. Collective excitations and the dynamical Stark effect in a coherently driven exciton system. Phys. Rev. Lett. 57, 2752-2755 (1986).

23. Lindberg, M. \& Koch, S. W. Effective Bloch equations for semiconductors. Phys. Rev. B 38, 3342-3350 (1988).

24. Pines, D. \& Nozieres, P. The Theory of Quantum Liquids (Benjamin, New York, 1966).

25. Bloch, F. Nuclear induction. Phys. Rev. 70, 460-474 (1946).

26. Schmitt-Rink, S., Mukamel, S., Leo, K., Shah, J. \& Chemla, D. S. Stochastic theory of time-resolved four-wave mixing in interacting media. Phys. Rev. A 44, 2124-2129 (1991).

27. Schäfer, W., Jahnke, F. \& Schmitt-Rink, S. Many-particle effects on transient four-wave-mixing signals in semiconductors. Phys. Rev. B 47, 1217-1220 (1993).

28. Axt, V. M. \& Stahl, A. A dynamics-controlled truncation scheme for the hierarchy of density matrices in semiconductor optics. Z. Phys. B 93, 195-204 (1994).

29. Maialle, M. Z. \& Sham, L. J. Interacting electron theory of coherent nonlinear response. Phys. Rev. Lett. 73, 3310-3313 (1994).

30. Östreich, T., Schönhammer, K. \& Sham, L. J. Exciton-exciton correlation in the nonlinear optica regime. Phys. Rev. Lett. 74, 4698-4701 (1995).

31. Schäfer, W., Lövenich, R., Fromer, N. A. \& Chemia, D. S. From coherently excited highly correlated states to incoherent relaxation processes in semiconductors. Phys. Rev. Lett. 86, 344-347 (2001).

32. Bolton, S. R., Neukirch, U., Sham, L. J., Chemla, D. S. \& Axt, V. M. Demonstration of sixth-order Coulomb correlations in a semiconductor single quantum well. Phys. Rev. Lett. 85, 2002-2005 (2000).

33. Meier, T., von Plessen, G., Thomas, P. \& Koch, S. W. Coherent electric-field effects in semiconductors. Phys. Rev. Lett. 73, 902-905 (1994).

34. Haring Bolivar, P. et al. Excitonic emission of THz radiation: experimental evidence of the shortcomings of the Block equation method. Phys. Rev. Lett. 78, 2232-2235 (1997).
35. Cohen-Tannoudji, C., Dupont-Roc, J. \& Grynberg, G. Atom-Photon Interactions: Basic Processes and Applications (Wiley Interscience, New York, 1992).

36. Kner, P. et al. Effect of magnetoexciton correlations on the coherent emission of semiconductors. Phys. Rev. B 60, 4731-4748 (1999).

37. Lepetit, L., Cheriaux, G. \& Joffre, M. Linear techniques of phase measurement by femtosecond spectral interferometry for applications in spectroscopy. J. Opt. Soc. Am. B 12, 2467-2474 (1995).

38. Birkedal, D. \& Shah, J. Femtosecond spectral interferometry of resonant secondary emission from quantum wells: resonance Rayleigh scattering in the nonergodic regime. Phys. Rev. Lett. 81, 23722375 (1998).

39. Kner, P., Schafer, W., Lovenich, R. \& Chemla, D. S. Coherence of four-particle correlations in semiconductors. Phys. Rev. Lett. 81, 5386-5389 (1998).

40. Banyai, L. et al. Exciton-LO-phonon quantum kinetics: evidence of memory effects in bulk GaAs. Phys. Rev. Lett. 75, 2188-2191 (1995).

41. Bar-Ad, S., Kner, P., Marquezini, M. V., Chemla, D. S. \& El Sayed, K. Carrier dynamics in the quantum kinetic regime. Phys. Rev. Lett. 77, 3177-3180 (1996).

42. Camescasse, F. X. et al. Ultrafast electron redistribution through Coulomb scattering in undoped GaAs: experiment and theory. Phys. Rev. Lett. 77, 5429-5432 (1996).

43. Bar-Ad, S. \& Chemla, D. S. Quantum kinetics regime during and immediately after laser excitation of semiconductors. Mater. Sci. Eng. B 48, 83-87 (1997).

44. Fürst, C., Leitenstorfer, A., Laubereau, A. \& Zimmermann, R. Quantum kinetic electron-phonon interaction in GaAs: energy nonconserving scattering events and memory effects. Phys. Rev. Lett. 78, 3733-3736 (1997).

45. Fromer, N. A. et al. Electronic dephasing in the quantum Hall regime. Phys. Rev. Lett. 83, 4646-4649 (1999).

46. Dodge, J. S. et al. Time-resolved optical observation of spin-wave dynamics. Phys. Rev. Lett. 83, 46504653 (1999).

47. Kaindl, R. A. et al. Ultrafast mid-infrared response of $\mathrm{YBa}_{2} \mathrm{Cu}_{3} \mathrm{O}_{7-\delta}$. Science $287,470-473$ (2000).

48. Garvin, S. M., McDonald, A. H. \& Platzman, P. M. Magneto-roton theory of collective excitation in the fractional quantum Hall effect. Phys. Rev. B 33, 2481-2494 (1986).

\section{Acknowledgements}

The work of D.S.C. was supported by the Director, Office of Science, Office of Basic Energy Sciences, Division of Materials Sciences and Office of Science, US Department of Energy.

Correspondence and requests for materials should be addressed to J.S (e-mail: jags@lucent.com). 